\section{Mechanisms compensating for visual field restriction in adolescents with damage to the retro-geniculate visual system}

L Jacobson ${ }^{1,2}$, F Lennartsson ${ }^{3,4}$, T Pansell' ${ }^{1}$, G Öqvist Seimyr ${ }^{2}$ and L Martin ${ }^{2,5}$

\begin{abstract}
Background To describe visual field (VF) outcome in three adolescents with damage to the optic radiation and to focus on mechanisms that may compensate the practical functional limitations of VF defects. Design Descriptive, prospective multi-case study in a hospital setting. Participants Three teenagers with cerebral visual dysfunction because of damage to the retro-geniculate visual pathways.

Methods Best-corrected visual acuity and eye alignment were assessed. Visual field function was tested with Goldmann perimetry, and with Rarebit, Humphrey Visual Field Analyzer and Esterman computerized techniques. Fixation was registered with video oculography during Rarebit examination. Magnetic resonance imaging of the brain illustrated brain damage and its relation to the posterior visual system.

Results One of the three subjects had bilateral asymmetric white matter damage of immaturity, early-onset exotropia, and a relative homonymous VF defect, but normal binocular VF. The second subject also had bilateral asymmetric white matter damage of immaturity and showed an inferior right quadrantanopia, confirmed by the binocular field. Registration of fixation revealed automatic scanning during perimetry. The third subject had an almost total left homonymous hemianopia after resection of a brain tumour in the right temporal lobe. The hemianopia could be compensated for by fast voluntary scanning.
\end{abstract}

Conclusion Congenital and later-acquired homonymous VF defects may, at least in young subjects, be compensated for by scanning. Exotropia may compensate VF defects and, therefore, the VF should be tested before strabismus surgery. Eye (2012) 26, 1437-1445; doi:10.1038/eye.2012.190; published online 21 September 2012

Keywords: VF defects; retro-geniculate visual system; compensating mechanisms; adolescents

\section{Introduction}

Brain damage has become the most common cause of visual impairment in children in developed countries ${ }^{1-3}$ as a consequence of improving survival of children with lifethreatening conditions with cerebral complications and of children born preterm. Assessment of visual function in these children may be hampered by their inability to understand or to co-operate in the tests. Therefore, there is still limited information available of visual outcome, and in particular of visual field (VF) function.

Brain imaging in children with cerebral visual impairment (CVI) reveals different pathologies of pre- or perinatal origin such as the following: malformations, white matter damage of immaturity, and cortico-subcortical damage (focal infarct, multicystic encephalomalacia, basal ganglia, and thalamic lesions). ${ }^{4}$ When brain damage occurs at 24-34-week gestation, the lesions typically occur in the white matter.
${ }^{1}$ Eye Unit, Department of Neuropaediatrics, Astrid Lindgren Children's Hospital, Karolinska University Hospital, Stockholm, Sweden

${ }^{2}$ Department of Clinical Neuroscience, Ophthalmology and Vision, Karolinska Institutet, Stockholm, Sweden

${ }^{3}$ Department of Medical Physics, Karolinska University Hospital, Stockholm, Sweden

${ }^{4}$ Department of Neuroradiology, Karolinska University Hospital, Stockholm, Sweden

${ }^{5}$ School of Health and Welfare, Mälardalen University, Eskilstuna, Sweden

Correspondence: Dr L Jacobson, Eye Unit, Department of Neuropaediatrics, Astrid Lindgren Childreńs Hospital, Karolinska University Hospital, Stockholm, SE 171 76, Sweden

Tel: +4686683510 E-mail: lena.jacobson@ karolinska.se or lena. jacobson@ki.se

Received: 9 May 2012 Accepted in revised form: 20 July 2012

Published online:

21 September 2012 
The specific lesions of the myelinated tracts comprise intraventricular haemorrhage, periventricular haemorrhagic infarction and periventricular leukomalacia. ${ }^{5}$ Each lesion may occur in isolation, but more commonly any single infant may present with multiple lesions. When a child is examined with cerebral imaging in childhood, the periventricular white matter pathology may be visualized. However, at that time, it may not be possible to know the cause. The end-stage lesion is described as white matter damage of immaturity, a common cause of CVI in prematurely born children. Later-acquired lesions due to trauma, stroke, tumour, or surgery may affect the posterior visual pathways and cause visual impairment. This heterogeneity of aetiologies representing insults at different developmental stages, may explain different prerequisites for reorganization and compensation, reflected in the VF outcome.

Exotropia as compensation in homonymous hemianopia has been suggested. ${ }^{6,7}$ Another compensatory mechanism based on saccadic exploration towards the blind field has been described. ${ }^{8}$ Motion detection in the blind field has also been described in adults with acquired hemianopia. ${ }^{9,10}$ With such compensation mechanisms, we can expect that the practical binocular VF function in everyday living differs from the monocular VF function assessed under strict control of fixation.

Training programs have been developed for adults with previously normal VF function who have acquired homonymous hemianopia, most often after stroke. These rehabilitation techniques are based on optical devices, on eye-movement training or visual restitution training. ${ }^{11}$ The value of such training programs is debated. ${ }^{12}$ No training programs have yet been designed for children with congenital or very early-acquired VF defects.

The practical consequences of VF defects in children with pre- and perinatal brain damage for mobility and orientation, for everyday life, for reading and for future driving, have not yet been thoroughly described.

The purpose of this multiple case presentation is to suggest possible automatically developed compensatory mechanisms that improve VF function in children with lesions affecting the posterior visual pathways because of pre- and perinatal or later-acquired brain damage.

\section{Subjects and methods}

Three teenagers with cerebral visual dysfunction because of the damage to the retro-geniculate visual pathways were recruited. Two had spastic cerebral palsy due to white matter damage of immaturity. One subject had undergone surgery for a brain tumour affecting the optic radiation in the temporal lobe.
All subjects were examined with Goldmann perimetry and three different types of computerized perimetry. Fixation and eye movements were registered during one of the computerized perimetries. Best-corrected decimal visual acuity was documented. Eye alignment was examined with cover test. Stereo acuity was tested with the TNO test (Laméris Ootech BV, Nieuwegein, The Netherlands), ${ }^{13}$ relying on the random-dot technique. ${ }^{14}$

The three boys and their parents gave consent before enrolment in the study. The study was approved by the Regional Ethics Committee, Stockholm North, in accordance with ethical standards on human experimentation and with the Helsinki Declaration of 1975, as revised in 1983.

\section{Perimetry}

Kinetic manual Goldmann perimetry was performed in a standardized way. ${ }^{15}$ The extent of the VF was determined using the II/4e stimulus. For the central VF, one or more of the stimuli I/3e, I/2, I/1e and, if possible, $0 / 1$ e were used. The fixation and co-operation during $\mathrm{VF}$ examinations of each subject were continuously carefully monitored and encouraged by the examiner. Every subject was prompted to fixate in the middle of the fixation mark, and every attempt to shift gaze led to renewed calls for looking straight ahead at the fixation mark. None of them had any obvious problems with shifting attention.

For quantitative measurement of the central VF, the Rarebit Perimetry was used, testing the $30^{\circ} \times 20^{\circ}$ central VF. The Rarebit perimetry technique ${ }^{16}$ tests the integrity of the retinal receptor matrix, using very small and bright dots against a dark background and has shown to be sensitive for damage in the visual system of different origins. ${ }^{16-19}$ The task for the subject is to follow a flickering fixation mark that moves over the computer screen and to indicate perception of the dots by a singleor a double-click on the computer mouse. Fixation stability is supervised by the examiner and encouraged by dynamically changing the fixation mark, ie, the fixation mark gradually diminishes in size, signalling a pending presentation. In order to cover a larger VF area, the fixation mark is moved according to a predefined pattern during the examination. The results are expressed as the mean hit rate, ie, the percentage of stimulus presented. Normal hit rates for adults have been found to range from 93 to 100\% (median 96\%), ${ }^{16,18}$ and are approximately the same for young subjects with a median mean hit rate of $93 \%$ (range 78-100) in 6.5-12year-old subjects and 97\% (range 89-100) in 14-20-yearold subjects. ${ }^{20}$ The results are also presented graphically, where unfilled rectangles indicate that all stimuli have been perceived in that area; the darker the rectangle, 
the more the stimuli that have been missed (see Figure 3). The learning effect is reported to be very low, $<1 \%$ unit in young subjects. ${ }^{21}$ The monocular VF examinations were performed with undilated pupils, and with appropriate correction for any refractive errors.

In addition, the Esterman test for functional scoring of peripheral vision, ${ }^{22}$ in the Humphrey Visual Field Analyzer, was used in subjects 1 and 2. The Esterman test is a binocular, suprathreshold test, where the stimuli are supposed to be strong enough, not to be much affected by refractive errors. Subject 2 also underwent an examination with standard automated perimetry (Humphrey Visual Field Analyzer; HFA (Carl Zeiss Meditec, Dublin, CA, USA)). All VF examinations were performed by a well-experienced perimetrist.

\section{Registration of fixation and eye motility}

In order to calculate any compensatory mechanisms, the C-ETD video oculography (Chronos Vision Inc., Berlin, Germany ${ }^{23}$ ) was used to record the right and left eye position during Rarebit perimetry by two miniature cameras. The head unit with the cameras was fixed to the head and thereby excluded unintended movements of the head unit. The eye positions were extracted from the recorded videos by the image software provided by the manufacturer of the system. The pupil position was used to calculate the horizontal and vertical eye positions. The temporal resolution of the video system was $200 \mathrm{~Hz}$ and the spatial resolution was $<0.1^{\circ}$. For comparison reasons, recordings of eye movements during Rarebit perimetry examination from one healthy adult subject are shown in Figure 1. The left recordings show the results when the subject is fixating and following the movement of the fixation mark accordingly. The right recordings show the results when the subject is instructed to scan for the stimulus. This reveals a scanning pattern with a relatively high frequency $(0.37 \mathrm{~Hz})$, with short lasting fixations $(0.32 \mathrm{~s}$ SD 0.14$)$,
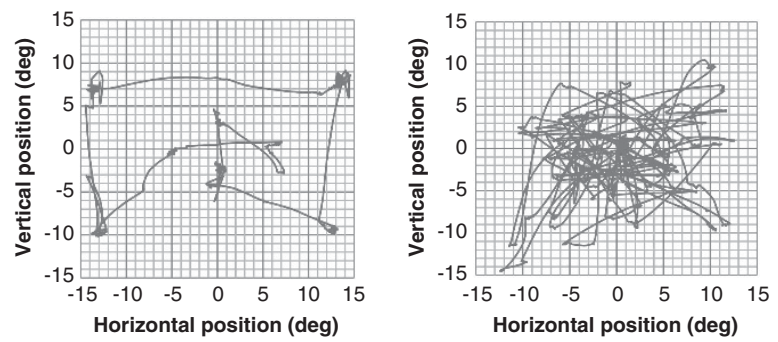

Figure 1 Healthy control subject: oculography during Rarebit perimetry examination of the left eye (left), when the subject was instructed and encouraged to fixate steadily at the moving fixation mark during the examination, and right eye (right), when the subject was instructed to scan for the stimulus. and a mixture of horizontal and vertical saccades (amplitude 6.1 deg SD 3.5) with no preferred direction.

\section{Magnetic resonance imaging}

Conventional structural MR images of the brain were acquired on a $1.5 \mathrm{~T}$ MR-system using the standard clinical protocols with T1- and T2-weighted and FLAIR images. In Subject 1, a high-resolution T1-weighted 3D volume was also collected. The primary rater was one of the authors (FL), and all cases were discussed with an experienced paediatric neuroradiologist from the department.

\section{Results and subject presentations}

Table 1 summarizes the results from the examinations in the current study.

\section{Subject 1}

This 17-year-old twin boy was born at the gestational age of 28 weeks. His neonatal period was complicated by intraventricular haemorrhage on the left side, and he was shunted because of hydrocephalus as an infant. He had an early-onset right exotropia and he was diagnosed with right-sided spastic cerebral palsy.

MRI revealed white matter damage of immaturity, consistent to severe bilateral periventricular leukomalacia, as a sequel to periventricular haemorrhagic infarction on the left side. There is severe reduction of the white matter volume in the mid and posterior left and in the posterior right hemisphere. The body and splenium of the corpus callosum are thinned and secondary degenerative lesions can be found in the left caudate, basal ganglia, thalamus, and brain stem with involvement of the cortico-spinal tract. There are no macroscopic lesions in the optic radiations and the lateral geniculate nuclei are spared bilaterally, but there is severe white matter reduction bilaterally and possible grey matter reduction in the left visual cortex (Figure 2, mid right).

Previous Goldmann perimetry had revealed an upper right homonymous defect for stimuli $\mathrm{I} / 2 \mathrm{e}$ and $\mathrm{I} / 1 \mathrm{e}$, and a subtle restriction for II/4e in the right hemifield. When the examination was done binocularly, all isopters within the $30^{\circ} \mathrm{VF}$ were normal (Figure 2, upper left), including the smallest and the weakest stimuli $(0 / 1 \mathrm{e})$, which the subject could not perceive monocularly, indicating an effect of binocular summation. ${ }^{24}$ However, a right inferior relative constriction was detected, which also was confirmed with the HFA (Figure 2, upper right). Rarebit perimetry showed a general sensitivity decrease in the whole central field in both eyes (Table 2 and 

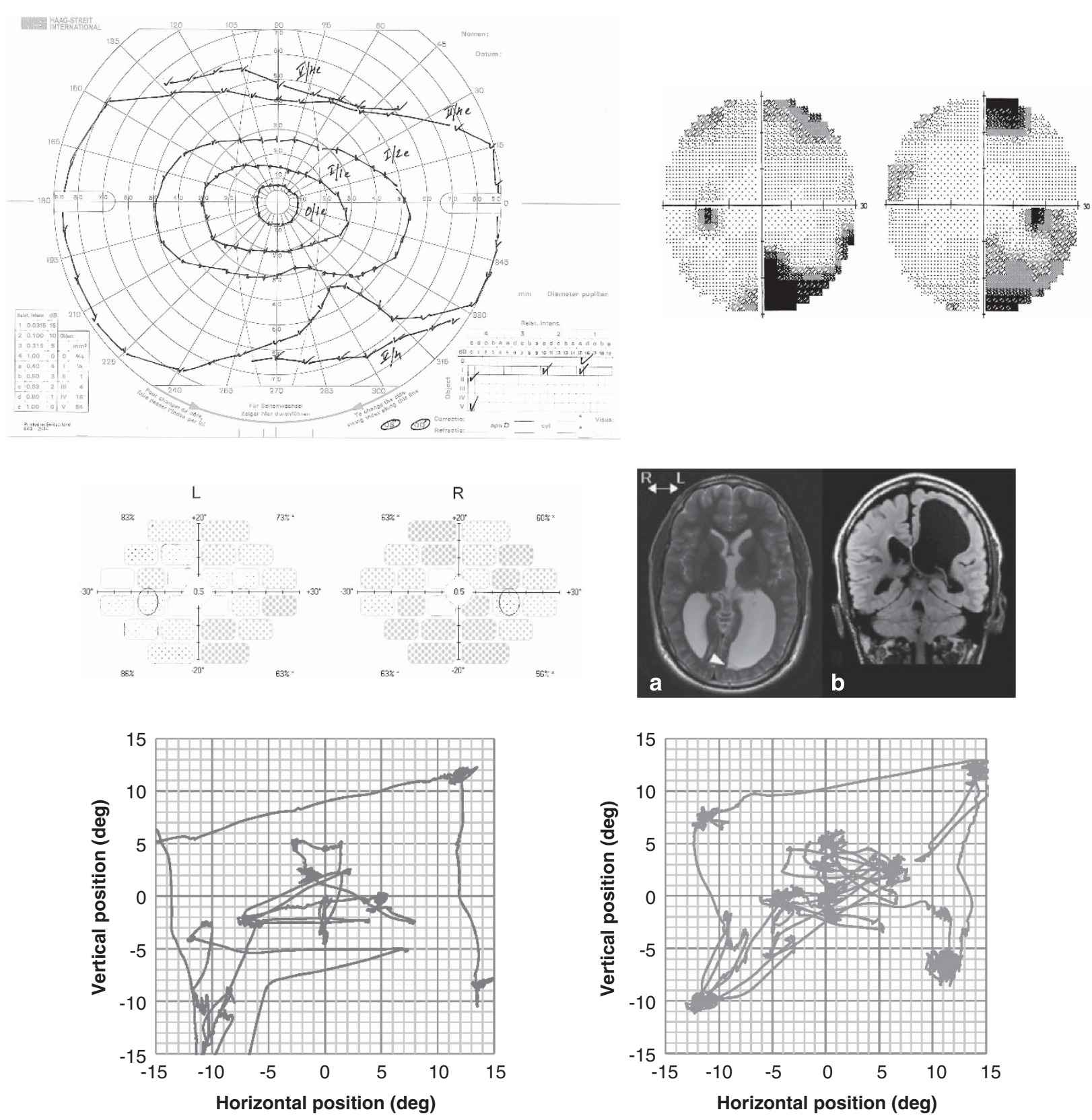

Figure 2 Subject 1 (MRI mid right) has severe white matter reduction in the posterior hemispheres and a pseudocyst in the posterior horn of the left lateral ventricle (arrow). The stratum sagittali have signs of gliosis and some normal appearing white matter in the inferior parts. Visual fields: upper left: binocular Goldmann visual field during fixation control; upper right: FA, RE (right eye) MD -6.55, LE (left eye) MD - 7.06; mid left: Rarebit perimetry RE 61\%, LE 76\% oculography during Rarebit perimetry examination of the LE and the RE (right eye); the subject was instructed and encouraged to fixate steadily during the examination. Note the instability during attempted fixation due to the nystagmus (compare with Figure 1), especially when fixating with the right eye.

Figure 2, mid left) and Esterman binocular suprathreshold perimetry, when the subject had both eyes open with a exo-deviation of $15-20^{\circ}$ in the right eye, showed a normal binocular field (Table 1).

The oculography during Rarebit perimetry examination showed a horizontal conjugate right beating nystagmus of $1.5^{\circ}$ in amplitude. The frequency is $\sim 1.6 \mathrm{~Hz}$ and the peak velocity of the fast phase below $100^{\circ}$ / sec (see Figure 2).

\section{Subject 2}

This 17-year-old boy was born at the gestational age of 27 weeks. He suffered from left intraventricular 
Table 1 Visual function, optic disc appearance and eye alignment

\begin{tabular}{|c|c|c|c|c|c|c|c|c|c|c|c|}
\hline \multirow[t]{2}{*}{ Subject } & \multirow[t]{2}{*}{ TNO } & \multicolumn{2}{|c|}{$\begin{array}{l}\text { Best-corrected } \\
\text { visual acuity }\end{array}$} & \multicolumn{2}{|c|}{ Disc area/cup area } & \multicolumn{2}{|l|}{ Strabismus } & \multicolumn{2}{|c|}{$\begin{array}{l}\text { Location of Goldmann } \\
\text { visual field defect }\end{array}$} & $\begin{array}{l}\text { Rarebit } \\
\text { visual field } \\
\text { defect }\end{array}$ & \multirow[t]{2}{*}{ Esterman $^{\mathrm{a}}$} \\
\hline & & Right & Left & Right & Left & Right & Left & Right & Left & Right Left & \\
\hline 1 & - & $6 / 12$ & $6 / 7.5$ & $\begin{array}{l}\text { Normal/ } \\
\text { small }\end{array}$ & $\begin{array}{l}\text { Normal/ } \\
\text { small }\end{array}$ & Exotropia & & $\begin{array}{l}\text { Inferior } \\
\text { temporal }\end{array}$ & $\begin{array}{l}\text { Inferior } \\
\text { nasal }\end{array}$ & $61 \% \quad 75 \%$ & $120 / 120$ \\
\hline 2 & 120 & $6 / 7.5$ & $6 / 6$ & $\begin{array}{l}\text { Normal/ } \\
\text { large }\end{array}$ & $\begin{array}{l}\text { Normal/ } \\
\text { normal }\end{array}$ & $\begin{array}{l}\text { Intermittent } \\
\text { exotropia }\end{array}$ & $\begin{array}{l}\text { Intermittent } \\
\text { exotropia }\end{array}$ & $\begin{array}{l}\text { Inferior } \\
\text { temporal }\end{array}$ & $\begin{array}{l}\text { Inferior } \\
\text { nasal }\end{array}$ & $89 \% \quad 91 \%$ & $112 / 120$ \\
\hline 3 & 60 & $6 / 6$ & $6 / 6$ & $\begin{array}{l}\text { Normal/ } \\
\text { normal }\end{array}$ & $\begin{array}{l}\text { Normal/ } \\
\text { normal }\end{array}$ & - & - & $\begin{array}{l}\text { Left } \\
\text { hemianopia }\end{array}$ & $\begin{array}{l}\text { Left } \\
\text { hemianopia }\end{array}$ & $57 \%$ n.e. & n.e. \\
\hline
\end{tabular}

asterman binocular visual field, dots seen/dots presented.

haemorrhage and was shunted as an infant because of hydrocephalus. He was diagnosed with bilateral asymmetric spastic cerebral palsy. MRI showed bilateral white matter damage of immaturity with periventricular leukomalacia and sequel after periventricular haemorrhage on the left side. There are white matter reductions in the mid and posterior hemispheres, with severe reduction on the left side. The body of the corpus callosum is thinned and there is a total agenesis of the splenium. There are secondary lesions in the left caudate, thalamus and brain stem but the basal ganglia are spared. The optic radiations are affected on both sides, most pronounced on left side with additional involvement of the lateral geniculate nucleus. The patient has an abnormally small left hemicranium (Figure 3, bottom right). He had an early-onset intermittent exotropia, but no nystagmus. Figure 3 shows the Goldmann VF and Rarebit perimetry, showing an inferior right quadrantanopia, and the binocular Esterman field, which could confirm the inferior defect.

Recordings of eye movements during Rarebit perimetry examination displayed short episodes of normal stable fixation, which were interrupted by shortlasting excursions from the fixation target, presumably due to searching for the stimulus. The scanning eye movements were seen primarily in the horizontal direction with an amplitude of 5.9 (SD 4.6) with a frequency of $0.7 \mathrm{~Hz}$ (see Figure 3, bottom left). The eye position was held still in fixations lasting for $0.48 \mathrm{~s}$ $(\mathrm{SD}=0.26)$. This boy was unaware of scanning.

\section{Subject 3}

This 15-year-old boy was diagnosed with a tumour in the right temporal lobe at 10 years of age. Before that, he had noticed VF loss during at least 1 year. At the time of diagnosis, he had a left homonymous hemianopia sparing a minor part of the hemifield, which was unchanged after surgery (Figure 4, upper).

MRI postoperatively showed a resection cavity in the medial right temporal lobe with surrounding fibrotic tissue. One year after the surgery, he was treated with radiotherapy in the area. The corpus callosum is unaffected but secondary lesions are found in the left thalamus and in the brain stem with atrophy in the pons. The right lateral geniculate nucleus and the first part of the optic radiation are disrupted and there are signs of secondary neurodegeneration in the optic radiation further downstream. The visual cortex appeared normal (Figure 4, mid right). The Rarebit perimetry showed an almost complete left hemianopia (mean hit rate 57\%) when he really forced himself not to scan, but to follow the fixation mark and otherwise keep the eye as steady as possible (Figure 4, mid left $\mathrm{R}$ eye). When told to behave as he normally does in real life, to explore the whole field during perimetry, the Rarebit perimetry showed a normal result (99\%) (Figure 4, mid left l eye).

The monitoring during Rarebit perimetry examination displayed normal fixation stability but showed a compensatory scanning technique. The scanning eye movements are primarily seen in the horizontal direction. The saccade amplitudes were on an average $3.5^{\circ}$ (SD 2.0) in amplitude and occurred on an average twice per second $(0.6 \mathrm{~Hz})$. Each eye fixation was held on an average $0.50 \mathrm{~s}$ (SD 0.30) see Figure 4, bottom. This boy was aware of scanning.

\section{Discussion}

These three teenagers with cerebral visual dysfunction represent different cerebral pathologies as follows: bilateral white matter damage of immaturity and a postoperative brain lesion after tumour. In the prematurely born subjects 1 and 2, the immature visual system was damaged neonatally; whereas in subject 3 , the tumour injured a more mature visual system at the approximate age of $8-9$ years.

Subject 1 has bilateral asymmetric white matter damage in the early third trimester. When analysing the MRI, we expected severe VF restriction. However, he has only relative homonymous defects with sparing of most of the outer limits of the VFs. These homonymous relative defects seem to be largely compensated for by 

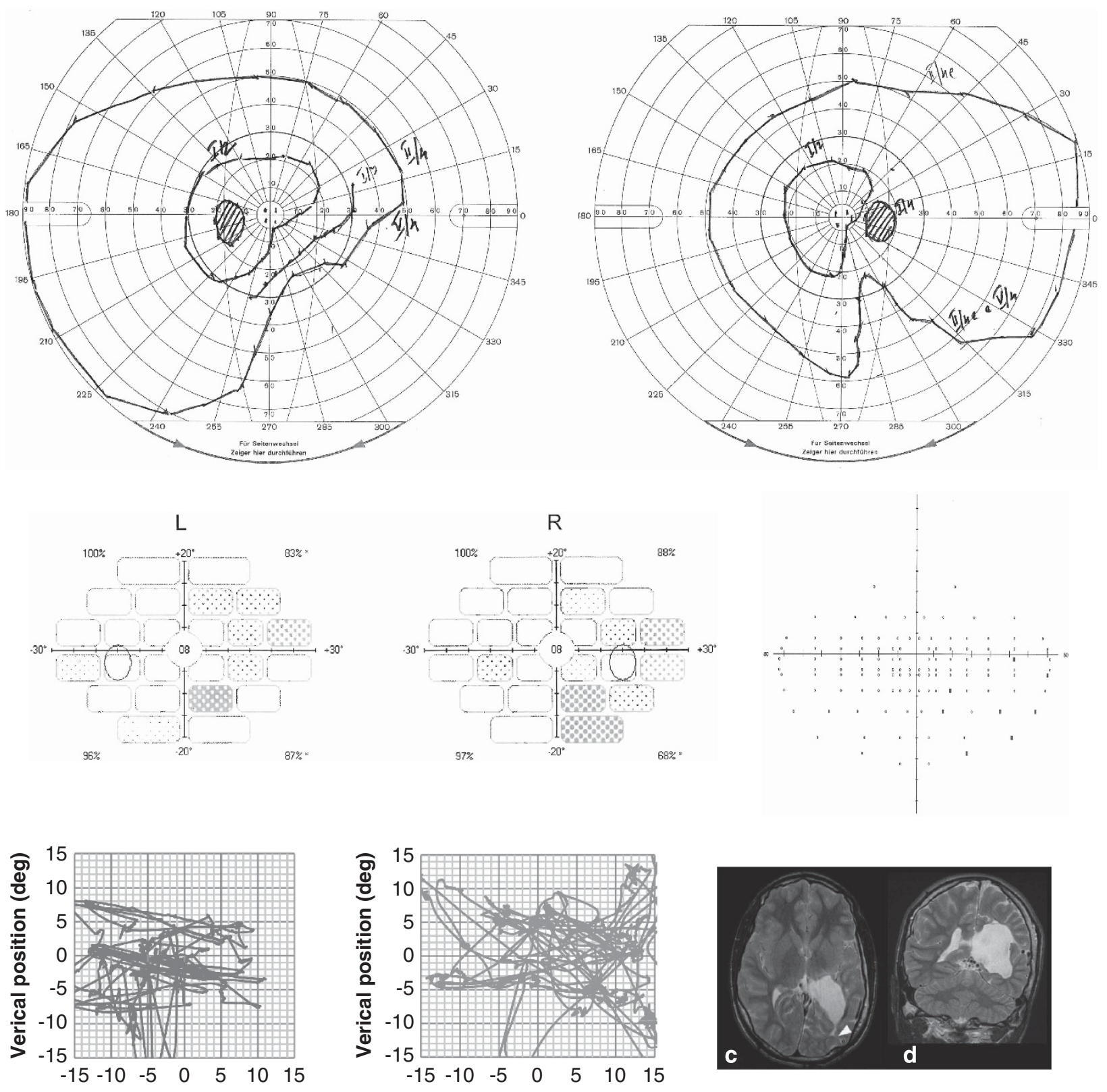

Horizontal position (deg)

Horizontal position (deg)

Figure 3 Subject 2 (MRI bottom right) shows severe affection of the optic radiation on the left side, and has a shunt from the posterior horn of the lateral ventricle (arrow). White matter reduction is seen in the stratum sagittale on the left side and in the superior part on the right side. Visual fields (upper): Goldmann visual fields, left and right eye; mid left: Rarebit perimetry right eye (RE) $89 \%$, left eye (LE) 91\%, unspecified defects in the right hemifields, most expressed inferiorly; mid right: Esterman binocular visual field, 112 scores out of 120, missed dots are in the right inferior quadrant. Oculography during Rarebit perimetry examination of the LE (bottom left) and the RE (bottom mid); the subject was instructed and encouraged to fixate steadily during the examination (compare with Figure 1).

exotropia $^{6,7}$ and the binocular VF function is found to be near normal when examined with binocular Goldmann perimetry and normal when examined with Esterman perimetry. As the exotropia in this subject seems to be of benefit for VF function, one could speculate that (cosmetic) strabismus surgery might be of great functional disadvantage in this case.
Also in subject 2, with bilateral white matter damage from early third trimester, a homonymous VF defect was found when examined with Goldmann perimetry.

However, on examination with Rarebit perimetry, with a fixation target that changes location on the screen during the procedure, he compensates by scanning, which he is not aware of. For the observer, this scanning resembles 

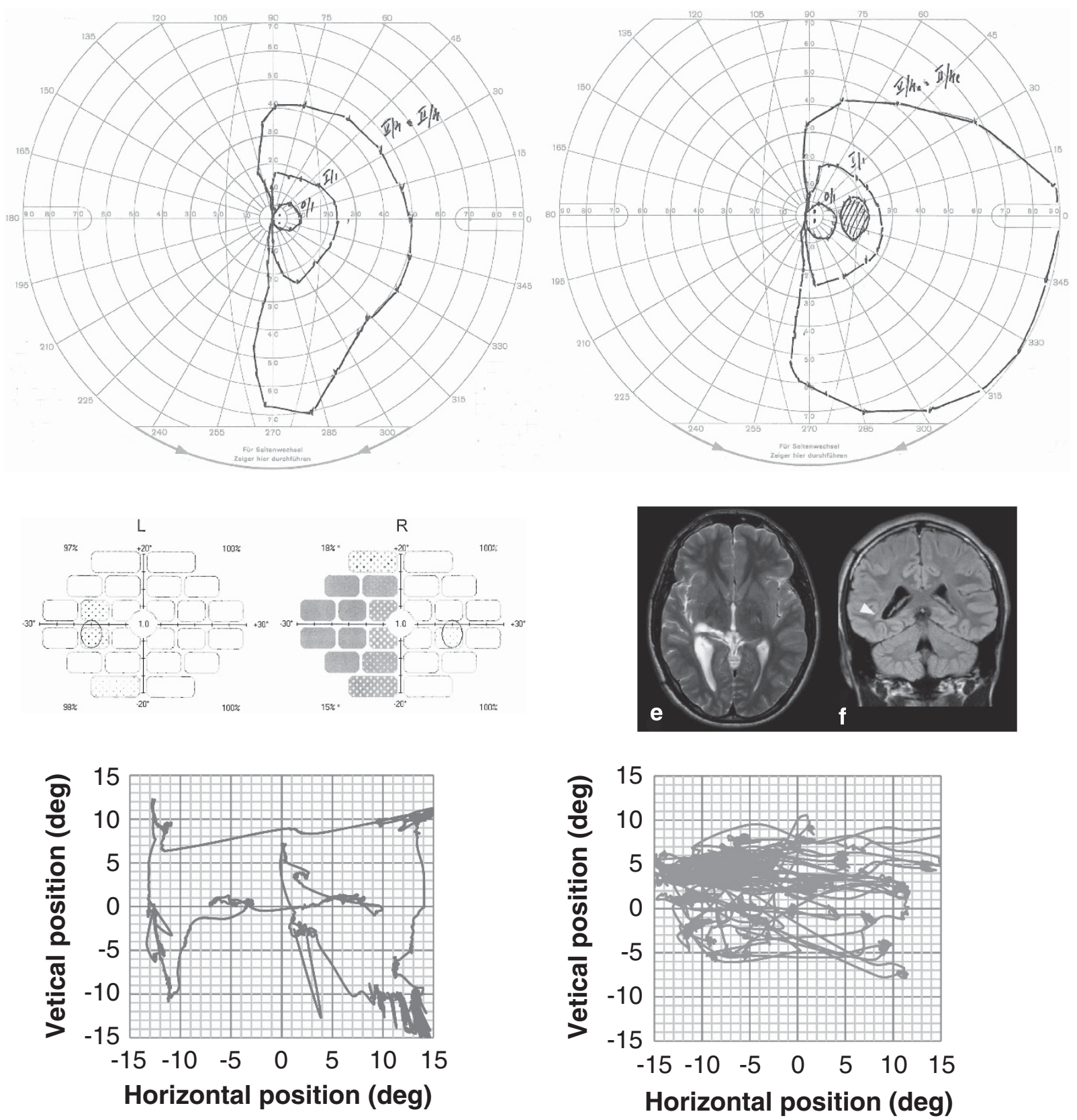

Figure 4 Subject 3 (MRI mid right) A resection cavity in the right medial temporal lobe disrupting efferent fibres from the lateral geniculate nucleus. Secondary neurodegeneration in the optic radiation in the right stratum sagittale (arrow).Visual fields; upper: Goldmann visual fields show left homonymous hemianopia; mid left: Rarebit perimetry right eye (RE) 57\% during strict fixation (mid left) and Rarebit perimetry RE 99\% during scanning (mid left). Oculography during Rarebit perimetry examination of the RE (bottom left), when the subject was instructed and encouraged to fixate steadily during the examination, and the left eye (LE) (bottom right), while behaving normally (compare with Figure 1).

nystagmus. During scanning, stimuli presented in the normal hemifield may be missed. Thus in this subject, the Rarebit perimetry does not give information about the 'neurological' VF, but provides important information about the 'functional' VF. Scanning, or saccadic exploration of the VF in hemianopia has previously been described. ${ }^{8}$
Subject 3 acquired a homonymous hemianopia later in childhood. He has developed an effective ability to scan, of which he is aware. One may speculate that his excellent performance in everyday life and as an elite player on the soccer field may be a consequence of scanning or of motion detection in the blind field ${ }^{9,10}$ or both. 
Subjects 2 and 3 both display a scanning eye movement pattern, which does not resemble the inappropriate saccadic intrusions often seen in patients with cerebellar disorders. The fixation duration was longer and the saccadic amplitudes larger than seen in patients displaying square-wave jerks, macrosaccadic oscillations, and ocular flutter. Neither does it resemble the voluntary scanning movements of the healthy subject in Figure 1. Our hypothesis is that these eye movements in subjects 2 and 3 fulfil a meaningful purpose and that they are not a result of a pathological condition.

For some individuals, the development of compensating mechanisms may occur naturally, maybe more effectively in the immature brain than in the adult, ${ }^{25}$ but certainly not for all. However, no evaluated programs have been developed to facilitate visual recovery in children with brain damage. Issues such as: if there is a critical period for intervention to promote visual recovery and how this intervention could be designed, still have to be explored.

\section{Conclusion}

Congenital and later-acquired homonymous VF defects may, at least in young subjects, be compensated for by scanning.

Pay attention to fixation during perimetry when studying VF outcome in adolescents with congenital or early-acquired brain damage. Automatic scanning, by the observer resembling nystagmus, may obscure the VF defect.

Exotropia may compensate for homonymous VF defects and, therefore the monocular and, in case of VF restriction, also the binocular VF should be tested before strabismus surgery.

\section{Summary}

What was known before

- Cerebral visual impairment (CVI) has become the most common cause of visual impairment in children.

- Exotropia and voluntary scanning to compensate hemianopia have been descibed in adults with acquired brain damage.

What this study adds

- Evidence for scanning as a compensating mechanism in young subjects with VF defects.

- The study illustrates the value of registering eye movements to assess fixation and scanning during perimetry.

\section{Conflict of interest}

The authors declare no conflict of interest.

\section{References}

1 Rogers M. Vision impairment in Liverpool: prevalence and morbidity. Arch Dis Child 1996; 74: 299-303.

2 Nielsen LS, Skov L, Jenson H. Visual dysfunctions and ocular disorders in children with developmental delay. I. prevalence, diagnoses and aetiology of visual impairment. Acta Ophthalmol Scand 2007; 85: 149-156.

3 Bunce C, Wormald R. Causes of blind certification in England and Wales: April 1999-March 2000. Eye 2008; 22: 905-911.

4 Bax M, Tydeman C, Flodmark O. Clinical and MRI correlates of cerebral palsy: the European cerebral palsy study. JAMA 2006; 4: 1602-1608.

5 Volpe JJ. Cerebral white matter injury of the premature child-More common than you think. Pediatrics 2003; 112: 176-180.

6 Herzau V, Bleher I, Joos-Kratsch E. Infantile exotropia with homonymous hemianopia: a rare contraindication for strabismus surgery. Graefes Arch Clin Exp Ophthalmol 1998; 226: $148-149$.

7 Donahue SP, Haun AK. Exotropia and face turn in children with homonymous hemianopia. J Neoroophthalmol 2007; 27: 304-307.

8 Zihl J. Visual scanning behaviour in patients with homonymous hemianopia. Neuropsychology 1995; 33: 287-303.

9 Henriksson L, Raninen A, Näsänen R, Hyvärinen L, Vanni S. Training-induced cortical representation of a hemianopic hemifield. J Neurol Neurosurg Psychiatry 2007; 78: 74-81.

10 Nelles G, Pscherer A, de Greiff A, Gerhard H, Forsting M, Esser J et al. Eye movement training-induced changes of visual field representation in patients with post-stroke hemianopia. J Neurol 2010; 257: 1832-1840.

11 Pambakian A, Currie J, Kennard C. Rehabilitation strategies for patients with homonymous visual field defects. J Neuroophthalmol 2005; 25: 136-142.

12 Schreiber A, Vonthein R, Reinhard J, Trauzettel-Klosinski S, Connert C, Schiefer U. Effect of visual restitution training on absolute homonymous scotomas. Neurology 2006; 67: 143-145.

13 Walraven J, Janzen P. TNO stereopsis test as an aid to the prevention of amblyopia. Ophthalmic Physiol Opt 1993; 13: 350-356.

14 Julesz B. Binocular depth perception without familiarity cues. Science 1996; 145: 356-361.

15 Sample P, Dannheim F, Artes P, Dietzsch J, Henson D, Johnson CA et al. Imaging and Perimetry Society Standards and Guidelines. Optom Vis Sci 2011; 88: 4-7.

16 Frisén L. New sensitive on abnormal spatial vision: rarebit probing. Vision Res 2002; 42: 1931-1939.

17 Martin L, Wanger P. New perimetric techniques: a comparison between rarebit and frequency doubling technology perimetry in normal subjects and glaucoma patients. J Glaucoma 2004; 13: 268-272.

18 Salvetat ML, Zeppieri M, Parisi L, Brusini P. Rarebit perimetry in normal subjects: test-retest variability, learning effect, normative range, influence of optical defocus and cataract extraction. Invest Ophthalmol Vis Sci 2007; 48: 5320-5331.

19 Hellgren K, Hellström A, Martin L. Visual field and optic disc morphology in very low birth weight adolescents examined with magnetic resonance imaging of the brain. Acta Ophthalmol Scand 2009; 87: 843-848. 
20 Martin L. Rarebit and frequency-doubling technology perimetry in children and young adults. Acta Ophthalmol Scand 2005; 83: 670-677.

21 Martin L, Aring E, Landgren M, Hellström A, Andersson Grönlund M. Visual fields in children with attentiondeficit/hyperactivity disorder before and after treatment with stimulants. Acta Ophthalmol Scand 2008; 86: 259-264.

22 Esterman B. Functional scoring of the binocular field. Ophthalmology 1982; 89: 1226-1234.

23 Clarke A, Ditterich J, Drüen K, Schönfeld U, Steineke C. Using high frame rate CMOS sensors for three-dimensional eye tracking. Behav Res Methods Instrum Comput 2003; 34: 549-560.

24 Wakayama MA, Matsumoto C, Ohmure K, Matsumoto F, Shimomura Y. Properties of receptive field on binocular fusion stimulation in the central visual field. Graefes Arch Clin Exp Ophthalmol 2002; 240: 743-747.

25 Tinelli F, Guzzetta A, Bertini C, Ricci D, Mercuri E, Ladavas $\mathrm{E}$ et al. Greater sparing of visual search in children after congenital rather than acquired focal brain damage. Neurorehabil Neural Repair 2011; 25: 721-728. 